\title{
Splicing mutation in Sbf1 causes nonsyndromic male infertility in the rat
}

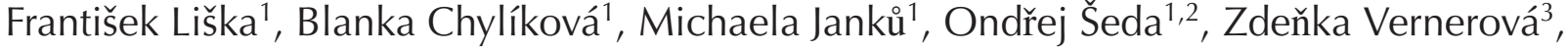 \\ Michal Pravenec ${ }^{1,4}$ and Vladimír Kř̌en ${ }^{1,4}$ \\ ${ }^{1}$ Institute of Biology and Medical Genetics, First Faculty of Medicine, Charles University in Prague, Prague 2, Czech \\ Republic, ${ }^{2}$ Laboratory of Rat Models of Metabolic Disorders, Division BIOCEV, Institute of Molecular Genetics of the \\ Academy of Sciences of the Czech Republic, Prague 4, Czech Republic, ${ }^{3}$ Institute for the Care of the Mother and \\ Child, Third Faculty of Medicine, Charles University in Prague, Prague 4, Czech Republic and ${ }^{4}$ Institute of \\ Physiology, Czech Academy of Sciences, Prague 4, Czech Republic
}

Correspondence should be addressed to F Liška; Email: frantisek.liska@lf1.cuni.cz

\begin{abstract}
In the inbred SHR/Olalpcv rat colony, we identified males with small testicles and inability to reproduce. By selectively breeding their parents, we revealed the infertility to segregate as an autosomal recessive Mendelian character. No other phenotype was observed in males, and females were completely normal. By linkage using a backcross with Brown Norway strain, we mapped the locus to a $1.2 \mathrm{Mbp}$ segment on chromosome 7, harboring 35 genes. Sequencing of candidate genes revealed a $\mathrm{G}$ to $\mathrm{A}$ substitution in a canonical 'AG' splice site of intron 37 in Sbf1 (SET binding factor 1, alias myotubularin-related protein 5). This leads to either skipping exon 38 or shifting splicing one base downstream, invariantly resulting in frameshift, premature stop codon and truncation of the protein. Western blotting using two anti-Sbf1 antibodies revealed absence of the full-length protein in the mutant testis. Testicles of the mutant males were significantly smaller compared with SHR from 4 weeks, peaked at $84 \%$ wild-type weight at 6 weeks and declined afterward to $28 \%$, reflecting massive germ cell loss. Histological examination revealed lower germ cell number; latest observed germ cell stage were round spermatids, resulting in the absence of sperm in the epididymis (azoospermia). SBF1 is a member of a phosphatase family lacking the catalytical activity. It probably modulates the activity of a phosphoinositol phosphatase MTMR2. Human homozygotes or compound heterozygotes for missense SBF1 mutations exhibit Charcot-Marie-Tooth disease (manifested mainly as progressive neuropathy), while a single mouse knockout reported in the literature identified male infertility as the only phenotype manifestation.

Reproduction (2016) 152 215-223
\end{abstract}

\section{Introduction}

Infertility is a widespread health problem, affecting approximately $8-12 \%$ couples (Agarwal et al. 2015) or about 70 million of couples worldwide (Boivin et al. 2007). Male factor is responsible for about half of the cases (Agarwal et al. 2015). Substantial proportion of male infertility cases remain idiopathic despite advances in clinical diagnostics; a large proportion of the latter is thought to be genetic in origin (Hotaling \& Carrell 2014, Krausz \& Chianese 2014). Most prevalent genetic cause of male infertility is Klinefelter syndrome, about $8 \%$ in azoospermic males (Vincent et al. 2002) and the azoospermia factor (AZF) deletions in the malespecific region of the $\mathrm{Y}$ chromosome, reaching $11 \%$ in azoospermic males (Foresta et al. 2001). Other genetic factors that cause spermatogenic failure in human are suspected, but their identification has been challenging. Study of genetically infertile animal models thus provided most of the information about genes required for proper spermatogenic function of the testis. According to the available models, most often targeted mutations in the mouse, azoospermia resulted from a block in germ cell development, most often in pachytene spermatocytes, but also in other stages of meiosis, in postmeiotic round spermatids and spermatogonia. Sertoli cell dysfunction is another reason for spermatogenesis failure with subsequent azoospermia (Liška 2003).

SBF1 (SET binding factor 1, SET being Su(var)3-9, Enhancer of zeste and Trithorax domain, also MTMR5) is a myotubularin family protein with catalytically inactive phosphatase domain. Highest level of SBF1 expression was observed in testis and central nervous system. In testis, it is expressed both in germ cells and in Sertoli cells (Firestein et al. 2002). SBF1 heterodimerizes with and stimulates the activity of MTMR2 (myotubularinrelated protein 2), also from the myotubularin family, but an active phosphoinositide lipid phosphatase, specific for position 3 in phosphatidylinositol-3monophosphate (Ptdlns3P) and phosphatidylinositol-3, 
5-bisphosphate (Ptdlns3,5P2). Ptdlns3P is in turn concentrated in early endosomes and is an important regulator of endosome trafficking (Laporte et al. 2003, Nicot \& Laporte 2008). Mice lacking SBF1 are viable, with isolated male infertility due to spermatogenesis failure resulting in azoospermia (Firestein et al. 2002). Human homozygotes or compound heterozygotes for damaging missense mutations develop Charcot-MarieTooth demyelinating peripheral neuropathy (Nakhro et al. 2013, Alazami et al. 2014). Here, we report a splicing site mutation leading to truncation of the rat SBF1 ortholog, which closely resembles the null mice with male infertility as principal pathological finding.

\section{Materials and methods}

\section{Animals}

Animal experiments were performed in conformity with the Animal Protection Law of the Czech Republic and were approved by the Ethical Committee of the First Faculty of Medicine, Charles University in Prague. Adult rat males were held under temperature- and humidity-controlled conditions on 12-h light:12-h darkness cycle. At all times, the animals had free access to food (standard chow) and water. Tail biopsy for DNA extraction was performed in local anesthesia. For tissue samples, animals were killed using isoflurane overdosing. The mutation was discovered in SHR/Olalpcv inbred rats and after isolation maintained by crossing the mutant females to heterozygous males. We named the mutation ifm (infertile males), Rat Genome Database assigned it identification number 10002782 and standard designation, after discovery of the gene mutation, $S b f 1^{m l l p c v}$.

\section{Linkage mapping}

Mutant homozygous ifm/ifm fertile females were crossed to BN/Cub wild-type males. F1 males were crossed to the mutant females to generate backcross progeny. DNA was isolated from tail biopsy using phenol-chloroform extraction and ethanol precipitation. Polymorphic microsatellites were amplified by PCR using primers with sequences retrieved from Rat Genome Database (RGD, http://rgd.mcw.edu/). Additional microsatellites for fine mapping were identified using Tandem Repeat Finder (Benson 1999) track in UCSC Genome Browser (http://www.genome.ucsc.edu/) and primers were designed using Primer 3 (Untergasser et al. 2012). The primers are listed in Supplementary Table 1, see section on supplementary data given at the end of this article. Standard PCR was performed on backcross population, using parental strains as controls. The PCR products were separated on agarose $(3-4 \%)$ or polyacrylamide $(7-10 \%)$ gels and detected in UV light after ethidium-bromide staining using Syngene G:Box (Syngene, Cambridge, UK).

\section{RT-PCR}

Testes were snap-frozen in liquid nitrogen and stored at $-80^{\circ} \mathrm{C}$. Tissue fragments were grinded in liquid nitrogen and the powder homogenized in Trizol (Invitrogen). After addition of chloroform, the water phase was applied to RNeasy columns (Qiagen) and purified according to manufacturer's instructions. Total RNA was converted to cDNA using SuperScript III reverse transcriptase and oligo-dT primer (Invitrogen). Specific primers for amplification and sequencing were designed by Primer3 and are listed in Supplementary Table 1.

\section{Microarrays}

We selected four control samples and four mutants aged 6 weeks. Double-stranded complementary DNA (cDNA) was synthesized from $50 \mathrm{ng}$ total RNA. The labeled and fragmented cDNA was hybridized to Affymetrix GeneChip Rat Gene 2.0 ST Arrays (Affymetrix). The whole hybridization procedure including DNA adjustment was performed according to the protocol recommended by Affymetrix.

\section{$q P C R$}

To validate microarray gene expression data, quantitative real-time PCR (SYBR-Green) was used. Total RNA $(1 \mu \mathrm{g})$ was reverse-transcribed with oligodT primers using the SuperScript III reverse transcriptase (Invitrogen). Primers were designed using Primer-Blast (Ye et al. 2012) or Primer3 (Supplementary Table 1). Real-time PCR reaction was performed in triplicate with EXPRESS SYBR GreenER qPCR SuperMix with Premixed ROX Kit according to the manufacturer's protocol (Invitrogen). Reactions were set up in 384 well plates using epMotion 5075 liquid handling workstation (Eppendorf, Hamburg, Germany). PCR amplification was performed using Applied Biosystems 7900HT Real-Time PCR System (Applied Biosystems). Each reaction contained cDNA amount corresponding to $5 \mathrm{ng}$ of initial total RNA. We used cyclophilin A (Ppia) as endogenous control.

\section{Statistical and pathway analyses}

Transcriptomic data: after evaluation of the hybridization, the quality control and the data normalization by robust multiarray analysis (RMA), the gene expression was compared between the SHR and the SHR ifm/ifm utilizing PARTEK Genomics Suite 6.6 (Partek, St. Louis, MO, USA). The transcripts found to be differentially expressed with raw $P$ value $<0.005$ and by more than $20 \%$ between the two groups were included in the gene enrichment and pathway analyses, which were performed using Ingenuity Pathway Analysis software (application build 364062M, content version 26127183). qPCR: statistical comparison was performed on $\mathrm{ddCt}$ values (normally distributed) by unpaired Student's t-test using Statistica 12 (StatSoft, Tulsa, OK, USA).

\section{Sequencing}

PCR or RT-PCR products were directly sequenced using BigDye Cycle Sequencing Kit v1.1 (Applied Biosystems). Multiple alternatively spliced RT-pcr products of mutants were first cloned into pCRII (Zero Blunt TOPO Cloning Kit, 
Invitrogen) and the clones sequenced using M13 primers and BigDye Cycle Sequencing Kit v1.1 (Applied Biosystems).

\section{Western blotting}

$\mathrm{N}$ - and C-terminal rabbit monoclonal SBF1 antibodies, EPR12566 and EPR12567 respectively were purchased from Abcam. Testis lysates were run on SDS-PAGE (8\% separating gel); proteins were blotted onto PVDF membranes Immobilon P (EMD Millipore). Membranes were incubated overnight at $4{ }^{\circ} \mathrm{C}$ with anti-SBF1 antibodies at final dilution 1:5000, secondary HRP-conjugated antibody was from GE Healthcare and signal was detected using ECL Prime chemiluminiscent detection kit (GE Healthcare). Control mouse monoclonal $\beta$-actin antibody was purchased from Abgent (San Diego, CA, USA).

\section{Histological examination}

After removal of tunica albuginea, testicular tissue was cut in cca $3 \times 3 \mathrm{~mm}$ pieces and fixed in Bouin solution. Standard procedures for paraffin embedding, sectioning and hematoxylin-eosin staining were employed. Slides were examined with a Nicon Eclipse E 600 light microscope (Nikon). All samples were evaluated in a blinded fashion. All microphotographs were made using Nicon NIS-Elements AR 3.1 morphometric software (Nikon). All samples were evaluated in a blinded fashion.

\section{Immunohistochemistry}

For immunohistochemical studies, $4 \mu \mathrm{m}$-thick paraffin sections were subjected to heat-induced epitope retrieval by immersion in an antigen retrieval solution (pH 6.0, Dako) for $30 \mathrm{~min}$ at $98^{\circ} \mathrm{C}$. Endogenous peroxidase was blocked by a 20-min treatment with $3 \%$ hydrogen peroxide in absolute methanol. Staining with rabbit monoclonal anti-SBF1 antibody (EPR12567, Abcam) diluted 1:800 in ChemMate antibody diluent (Dako) was performed for $1 \mathrm{~h}$ at room temperature. Staining with rabbit polyclonal anti-SCF1 antibody (C-Terminus) (LS-C2911, LSBio, Seattle, WA, USA) diluted 1:400 in ChemMate antibody diluent (Dako) was performed for $1 \mathrm{~h}$ at room temperature. The Envision Kit (Dako) was used to visualize sections incubated with primary antibodies. The chromogen 3,3-diaminobenzidine was applied to all sections and counterstaining was performed with Mayer's hematoxylin. Optimal staining conditions of any given antibody were determined using appropriate positive and negative controls.

\section{Results}

\section{ifm is mapping to rat chromosome 7}

In our inbred spontaneously hypertensive rat (SHR/ Olalpcv) colony, several males from the same family were unable to reproduce and had strikingly small testicles. By breeding the parents and sisters, we were able to isolate the trait and ascertain that it was inherited in Mendelian fashion, showing autosomal recessive pattern. Only males were infertile; there was no discernible phenotypic abnormality in females. We named the mutation ' ifm'. To ascertain the fertility of the ifm males, we cohoused $15 \mathrm{ifm} / \mathrm{ifm}$ males with wild-type females (who were proven fertile with wild-type males) for at least 1 month. Despite normal sexual behavior of the males, we did not observe any pregnancy.

Using 54 backcross males, we performed genetic mapping of the mutation with 212 microsatellite polymorphisms distributed over the 20 rat autosomes, and we revealed linkage of the mutation to rat chromosome 7. Fine mapping with additional 325 males specified a nonrecombinant region comprising 1.2 Mbp (coordinates chr7:127047128-128250751, rat genome Baylor version 3.4, Fig. 1A). The region contains 34 protein coding and one miRNA gene. We excluded most of the genes as functional candidates based on the available information about their expression pattern and molecular function. Therefore, we sequenced four 'functional candidate' genes with possible involvement in spermatogenesis and male fertility: Mov10l1, Sbf1, Odf3b and Syce3 using testicular cDNA. Mov10/1, Odf $3 b$ and Syce 3 coding sequences did not contain any sequence variation between SHR and ifm homozygotes.

\section{Sbf1 contains a mutation in acceptor splice site}

Amplification of cDNA from testes of the mutants with primers flanking exon 38 (SBF1 structure see Fig. 1B) resulted in altered band pattern compared with the expected product size amplified from control cDNA. The altered pattern was also observed in heterozygotes, albeit with lower intensity. Sequencing of the mutant RT-PCR products (74 clones) recovered eight variants with different splicing (Fig. 1C). There was, in general, either skipping of exon 38 or retainment of intron 37. In products with 'correct' splicing of intron 37 and exon 38 first base of exon 38 was missing. All these events are causing frame shifting and are predicted to result in SBF1 protein truncation. The reason for the aberrant splicing, as we uncovered by sequencing genomic DNA, was transition from $G$ to $A$ in position -1 of intron 37 (Fig. 1D), disrupting the canonical AG acceptor splice site. Consequently, as the first base of the exon 38 is G, a new acceptor splice site was created just 1 bp downstream compared with wild type. The new splice site was less efficient, as predicted by lower NNSPLICE score (Reese et al. 1997, Fig. 1D) and resulted in increased frequency of failure to splice out intron 37 or skipping exon 38. Expression level of Sbf1 mRNA was significantly lower in mutant testes, but also in some other organs, presumably due to nonsense-mediated decay of transcripts containing premature stop codons (Supplementary Fig. 1A). Sensitive qPCR assay identified SBF1 expression in all tested organs; however, Western blotting with C-terminal SBF1 antibody revealed fulllength SBF1 expression in brain, adrenals and male reproductive tract; weak expression was also observed 
A

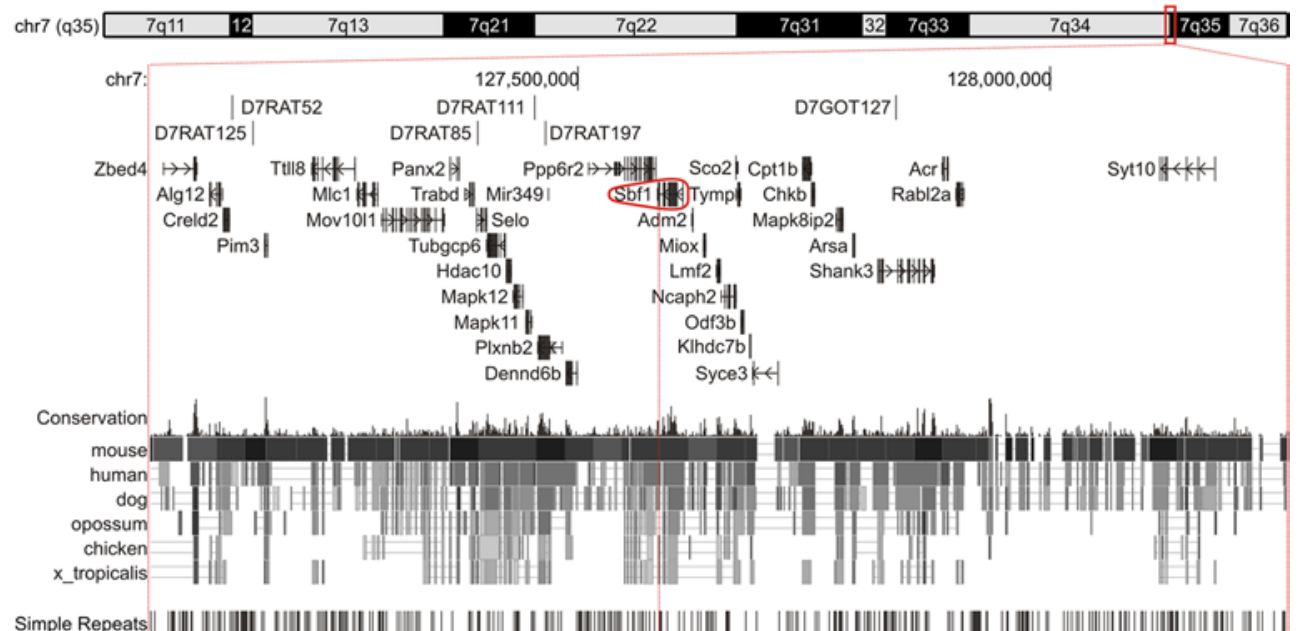

B

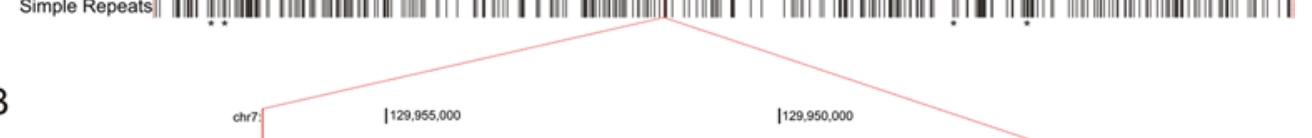

C

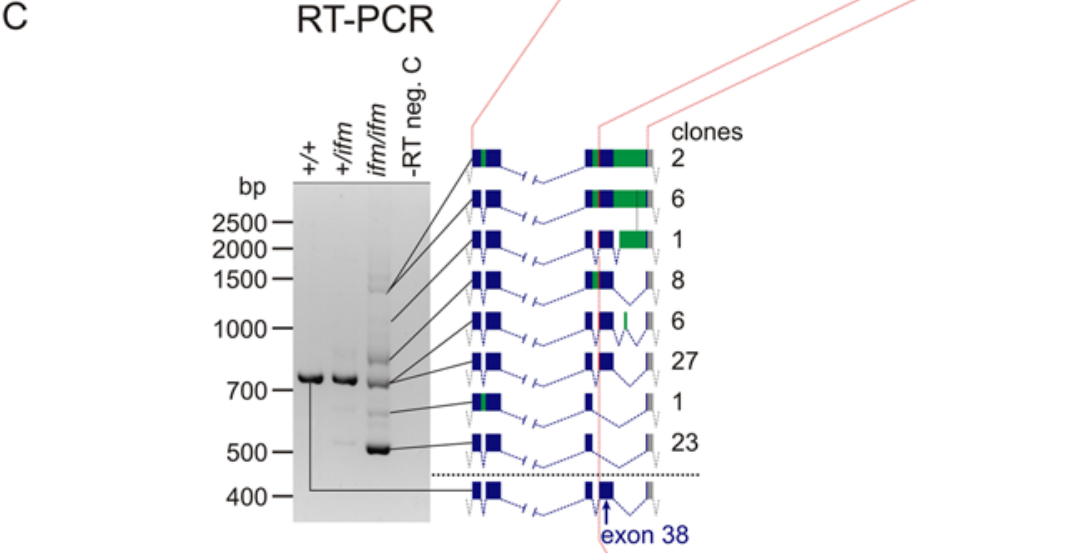

D
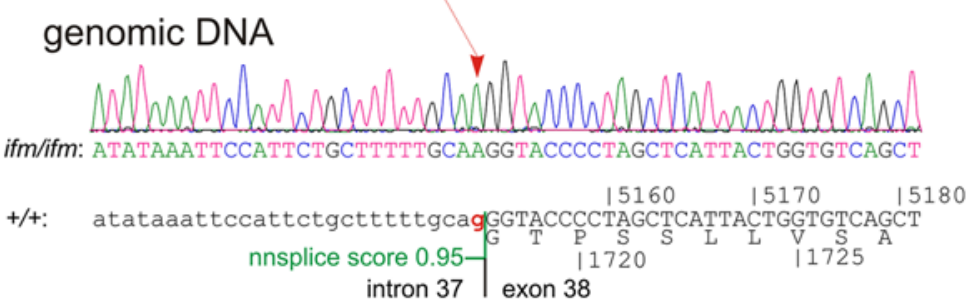

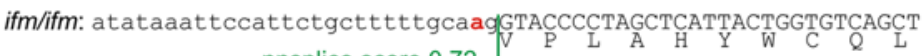

nnsplice score 0.72

\section{c.5152_-1G>A .. p.G1718fs X1745}

Figure 1 (A) Nonrecombinant region of mutation on chromosome 7. Map is based on UCSC Genome Browser view, rat genome version 3.4. Top track - all polymorphic markers that manifested complete linkage with the mutant allele. Middle - genes in the region, SBF1 encircled. Bottom conservation and simple repeats; asterisks highlight positions of additional completely linked 'custom' microsatellite polymorphisms. (B) Structure of $3^{\prime}$ portion of Sbf1 gene and position of the mutation at the intron 37/exon 38 boundary (middle red vertical line). Left and right red vertical lines depict forward and reverse primer positions respectively. (C) RT-PCR of mutant testis RNA demonstrates multiple aberrant splicing events with either skipping exon 38 or retaining some of the introns. Aberrant spliced-in introns are in green, standard exons in blue. The number on right represents the number of cDNA clones sharing identical structure from total 74 clones. (D) Sanger sequencing electropherogram showing the intron 37 mutation c.5152_-1G>A. This shifts the acceptor splice site one nucleotide downstream, resulting in frameshift at protein position 1718 and premature termination codon at position 1745 (p.G1718fsX1745). Additionally, NNSPLICE score for each splice site is shown in green. 
in kidneys and liver (Supplementary Fig. 1B). Using immunohistochemistry (IHC), we confirmed SBF1 expression in testes (see below) and brain, where we observed granular cytoplasmic positivity of SBF1 within neurons (Supplementary Fig. 2A and B), In agreement with Western blotting experiments, we additionally observed similar granular cytoplasmic positivity in kidneys - within the tubular compartment, no staining was apparent in glomeruli (Supplementary Fig. 2C and D). Heart and liver did not express SBF1 by IHC. However, nonspecific positivity within the endothelium was present in all organs.

\section{ifm/ifm homozygotes lack full-length Sbf1 protein}

Western blotting of lysates from testes of the mutant homozygotes compared with wild type using the C-terminal antibody (Fig. 2A) revealed the lack of full-length SBF1 protein (expected molecular weight $211 \mathrm{kDa}$, apparent molecular weight $220 \mathrm{kDa}$, Fig. 2B). Interestingly, the C-terminal antibody also detected some lower molecular weight species in both mutant and wild-type samples. These protein bands may result from alternative transcripts skipping the mutated splicing site inframe. Such transcripts are theoretically possible; however, by analysis of 74 cDNA clones, we found all transcripts to contain frameshift upstream of the antibody binding site. It is, therefore, more likely that the additional bands likely represent cross-reactivity with other proteins. Using N-terminal antibody, we confirmed the lack of full-length SBF1 in mutant homozygotes
(Fig. 2C). This antibody also detected additional lower molecular weight protein bands. One of them, around $210 \mathrm{kDa}$, was also detected by the C-terminal antibody, making it more plausible that the protein band represents real SBF1 variant expressed also in mutants.

\section{Azoospermia in ifm/ifm males}

Testis weight in mutant homozygotes falls behind the wild type at 4 weeks, peaks at 6 weeks (still below wild type), then drops down to one-third to one-quarter of wild type in 4-month adults (Fig. 3A). Epididymides of the mutants were devoid of any sperm; the rats were fully azoospermic.

\section{Blocked spermiogenesis in ifm/ifm males}

During the postnatal development, first evidence of abnormality is slightly lower cellularity of the tubules accompanied by vacuolization of the Sertoli cells (32 days, Fig. 4A and B). In adults, the mutant testes did not contain elongated spermatids (60 days, Fig. $4 \mathrm{C}$ and D), only diminished numbers of round spermatids could be detected (arrow in Fig. 4D). Lower amount of germ cells and enlarged vacuoles of Sertoli cells were evident (Fig. 4D and F compared with $\mathrm{C}$ and $\mathrm{E}$ ).

\section{Differential gene expression in ifm/ifm males}

We performed transcriptome analysis in testes of 6-week-old Sbf1 mutant homozygotes and wild-type

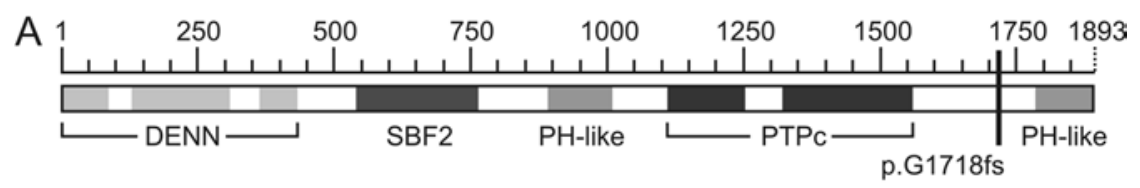

anti-Sbf1 N-terminal antibody

B

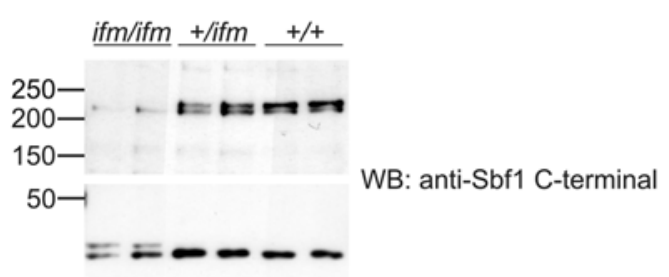

$35-$

C 250-

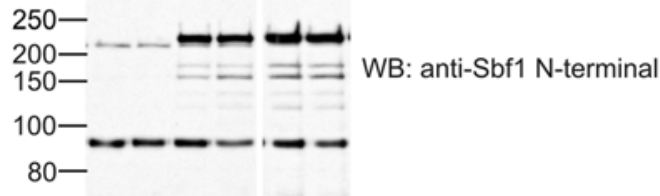

D

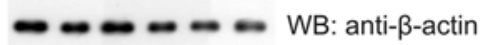

Figure 2 (A) Domain structure of Sbf1 protein as retrieved form $\mathrm{NCBI}$-conserved domain database (Marchler-Bauer et al. 2015). Compare also with (Laporte et al. 2003). Mutation ifm leads to loss of C-terminal pleckstrin homology domain. (B) Western blotting of testicular lysates using C-terminal SBF1 antibody (see A). Full-length Sbf1 (apparent molecular weight approximately $220 \mathrm{kDa}$, expected molecular weight $211 \mathrm{kDa}$ ) is completely missing in mutants. However, the antibody also detects shorter species (210, 45 , and $43 \mathrm{kDa}$ ) in all samples. They may represent other cross-reacting proteins. (C) Western blot using N-terminal Sbf1 antibody confirms the lack of full-length Sbf1 protein in mutants. The shorter $210 \mathrm{kDa}$ species is also detected by this antibody. Additionally, a $90 \mathrm{kDa}$ band is visible in all samples. (D) $\beta$-actin was used as endogenous control. 

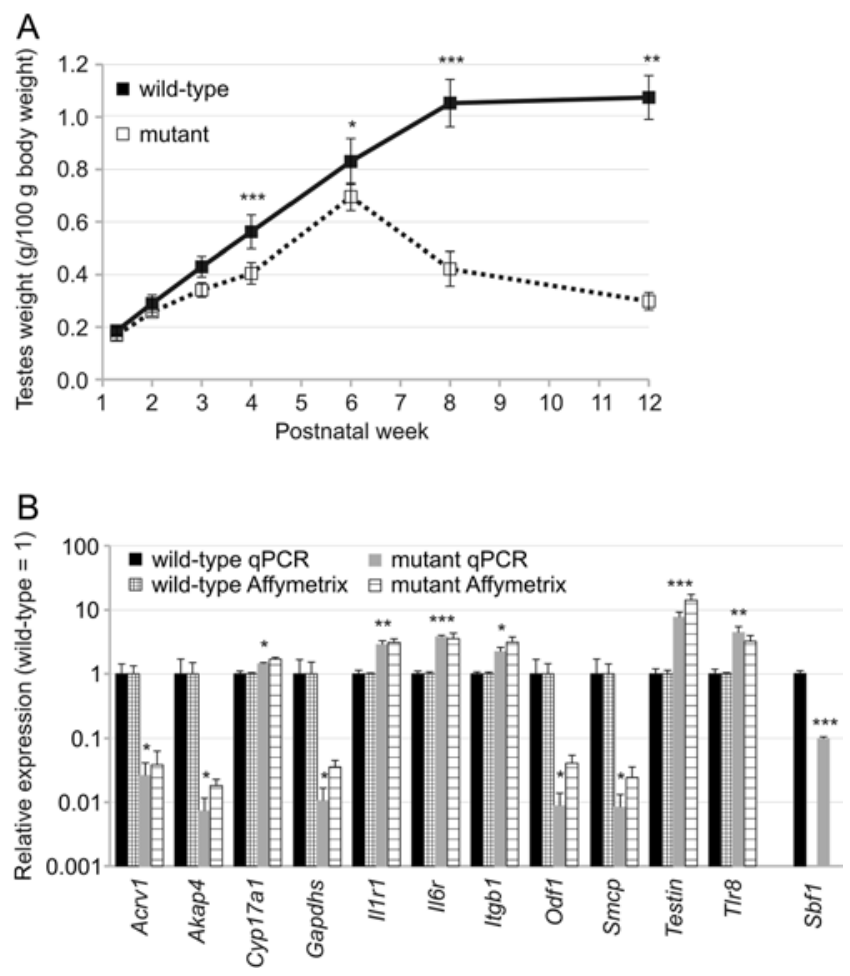

Figure 3 (A) During the first wave of spermatogenesis, the testis weight in mutants grew on a lower level compared with wild-type SHRs. After a peak at 6 weeks of age, testis weight declined sharply, reflecting massive germ cell loss. In the wild-type SHR, testis weight reached plateau in at the same time $(8-12$ weeks). Data are mean \pm S.D. Minimum number of animals per group was 3. Statistical significance: ${ }^{*} P<0.05,{ }^{* *} P<0.01,{ }^{* * *} P<0.001$ (Student's $t$-test with Bonferroni correction for multiple comparison). (B) Genes differentially expressed between wild-type and Sbf1 mutant testes. Data represent means, error bars standard errors. Wild-type expression was set to 1 . Both microarray and qPCR values are depicted, with the exception of Sbf1, which was not represented in the rat microarray.

controls ( $n=4$ for each group) using Affymetrix microarrays. We selected that age since the first wave of spermatogenesis should already produce haploid spermatids while the seminiferous epithelium was not yet degenerated. 494 transcripts had raw $P$ values $<0.005$ and 308 corresponded to known genes (Supplementary Table 2 for all known genes with raw $P$ values $<0.005)$. The comparison did not reach wholegenome significance using Benjamini-Hochberg correction; however, we were able to confirm 11 genes to be differentially expressed using qPCR (Fig. 3B). Genes expressed in spermatids were strikingly downregulated in mutants compared with wild type; these included Acrv1 (Golden et al. 1993), Akap4 (Brown et al. 2003), Gapdhs (Welch et al. 1995), Odf1 (Yang et al. 2012), Smcp (Nam et al. 1997) and many other genes not validated by $\mathrm{qPCR}$, confirming the histopathological findings. Conversely, at least some Sertoli cell-specific
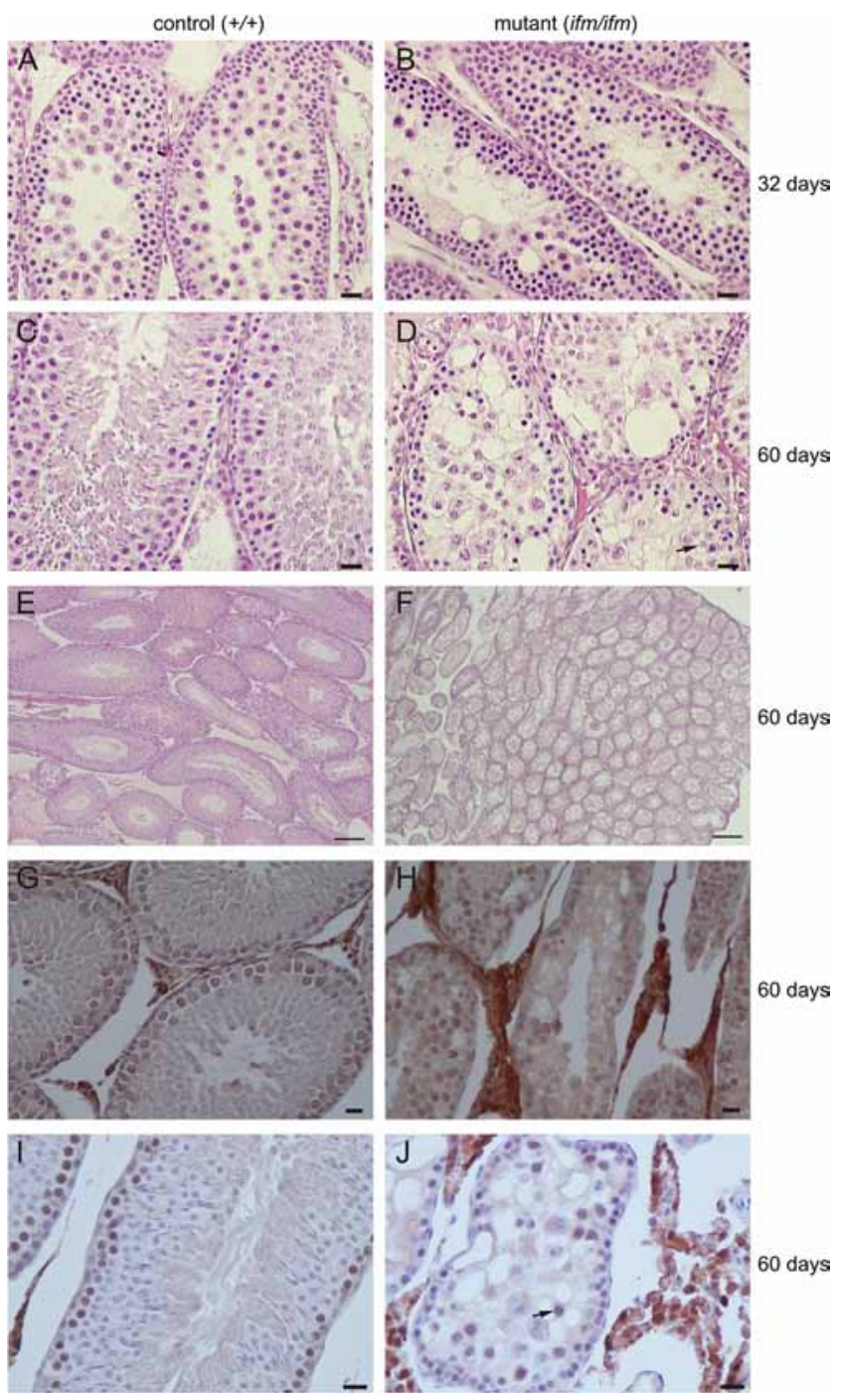

Figure 4 Histological examination of testis. (A and B) Juvenile seminiferous tubules of ifm/ifm homozygotes (32 days, B) show lower cellularity and vacuoles formed in Sertoli cells compared with wild type (A). (C and D) Mature tubules also demonstrate complete absence of elongated spermatids in mutants (D) compared with wild type (C). Spermatogenesis is blocked in round spermatid stage, resulting in azoospermia (absence of sperm in the epididymis, not shown). (E and F) Low magnification of samples C + D. Sertoli cell vacuoles are prominent, germ cells strikingly reduced. ( $\mathrm{G}$ and $\mathrm{H}$ ) Immunohistochemical detection of Sbf1 in testis using the C-terminal antibody. Highest expression was observed in basal layer of the seminiferous epithelium comprising spermatogonia, and in primary spermatocytes. Mutants show diminished signal in the seminiferous epithelium. High-density signal in the interstitial compartment is not specific (as it was observed in negative control preparations). Note hypertrophy of the interstitial (Leydig cell) compartment in mutants. (I and J) Immunohistochemical detection of SCP1. Control sample shows nuclear positive signal in primary spermatocytes in second from basal layer of the seminiferous epithelium. In mutants, positive spermatocytes in prophase of meiosis I are also observed, but they are situated also in the adluminal compartment, as the epithelium is disorganized (arrow). Scale bars $20 \mu \mathrm{m}$, in panels E and F $250 \mu \mathrm{m}$. 
transcripts were upregulated, from which we validated Testin (Cheng et al. 1989) and Itgb1 (Mulholland et al. 2001, Beardsley et al. 2006). The reason of Sertoli cellspecific transcript upregulation could be the relative increase in Sertoli cell compartment due to missing spermatids in mutants. In testin case, it can be also due to a lacking feedback from germ cells (Cheng et al. 1989). $\beta 1$-integrin (coded by Itgb1) upregulation can point to impairment of the ectoplasmic specializations between Sertoli cells and (diminished) germ cells. A modest increase (1.4-fold according to gPCR) in steroidogenic enzyme 17 $\alpha$-hydroxylase/C17-20 lyase transcript Cyp17a1 (Payne \& Youngblood 1995) can indicate an increased proportion or hyperplasia of Leydig cells. Another group of genes upregulated in mutants represented molecules involved in inflammatory response: T/r8 (Dasari et al. 2008), Il6r (Rival et al. 2006) and I/1r1 (Lysiak 2004). Genes specifically expressed during different developmental stages of spermatogonia (such as Id4, Cdh1, Zbtb16, Kit) were not differentially expressed (raw $P$ values $>0.05$ ). Similarly, there was no difference between ifm mutants and wildtype controls in the expression of meiosis-specific or primary spermatocyte genes (e.g. Scp2, Prdm9, Rad51, Brca1, Brca2). Ingenuity Pathway Analysis also did not indicate enrichment of apoptosis genes in the 308 known putatively differentially expressed genes (Supplementary Fig. 3).

\section{Immunohistochemical analysis of SBF1}

To confirm the SBF1 expression at the protein level and explore its distribution pattern, we examined Sbf 1 by IHC in testes. Strong cytoplasmic positivity was observed in spermatogonia, pachytene sprematocytes, Leydig and Sertoli cells during normal spermatogenesis at different postnatal days. We found only weak cytoplasmatic positivity in postmeiotic spermatids. Interestingly, the SBF1 signal was not absent in samples from mutants, only somewhat weaker and wild-type rats exhibited more positive spermatogonia compared with mutants (Fig. 4G and $\mathrm{H}$ ). This finding is consistent with the possibility that minor alternative forms of SBF1 protein are still expressed in mutants, as suggested by Western blots.

We used antibody against SCF1 (major component of the transverse filaments of synaptonemal complexes) as a marker of primary spermatocytes to confirm their presence in mutant males. As expected from the lack of differential expression of spermatocyte-specific genes, primary spermatocytes were found in mutants. However, they were not retained in the second to basal compartment of the seminiferous epithelium like in controls, but were found also closer to the adluminal compartment, suggesting impairment of the germ cell-Sertoli cell junctions (Fig. $4 \mathrm{I}$ and J).

\section{Discussion}

Targeted Sbf1 null mutation was reported (Firestein et al. 2002). The mice lacking Sbf1 exhibited failure of spermatogenesis and azoospermia. The mutation described here recapitulates all major findings of the null mouse, namely the vacuolization of the Sertoli cells as the first histopathological sign, progress of the first wave of spermatogenesis to round spermatid stage, but not further, and progressive degeneration of the germ cells in older animals. The rat mutation can be predicted as null too, due to the splicing disruption and protein truncation predicted from transcription data. However, the mutant transcripts are present, although the expression level is lower. Moreover, the Western blotting and IHC experiments suggest possible presence of non-full-length or truncated SBF1 variants in mutants. The functional impact of these shorter SBF1 variants is doubtful, e.g. observed normal phenotype of heterozygotes would be less likely in presence of a truncated protein that could disrupt the protein complexes, presumably MTMR2/Sbf2, that require SBF1. It was shown recently that human homozygotes or compound heterozygotes for missense SBF1 mutations exhibit hereditary progressive neuropathy CharcotMarie-Tooth disease type 4B3 (Nakhro et al. 2013, Alazami et al. 2014). There is no neurological impairment even in old SHR ifm/ifm rats, nor there was reported any such phenotype for the knockout mice (Firestein et al. 2002). It remains to be investigated, whether there is any subclinical neurological impairment in the rat or mouse mutants. In the rat mutation described here, it could be a compensatory effect of the shorter SBF1 variants still produced in mutants. On the other hand, the point mutations described in CMT4B3 patients may exert a different mode of action, i.e. they can represent 'gain of function'. Since it can be predicted that the proteins are expressed and could potentially bind to their interaction partners, they can block the normal function of the respective protein complexes. In human unaffected heterozygotes, some of the complexes may be normal, depending on stoichiometry, which may be enough for phenotype compensation. In the null mice (or mutant rats), there could also be a compensation achieved by different member of SBF family, like SBF2 (MTMR13) in neurons but not in male germ cells. Strikingly, Mtmr2 knockout mice develop CMT4B and azoospermia (Bolino et al. 2004); however, in male cases of CMT4B3 with Sbf1 mutations, spermatogenesis was not evaluated (Bohlega et al. 2011) leaving open the possibility that it could be also abnormal. Despite forming a complex, MTMR2 and SBF1 functions in spermatogenesis may not be identical; there is a phenotype difference between Mtmr2 mutant mice whose major pathology seems to be shedding of germ cells into lumen, which is not observed in Sbf1 mutants. Although it is not known whether Sertoli cell function or germ cell function of SBF1 is required 
for spermatogenesis, some mouse mutants which disrupt genes believed to be Sertoli cell-specific have some phenotypic resemblance to the SBF1 mutation (Liška 2003). Sertoli cell involvement can be also supported by a speculative mechanism, involving the upregulated inflammatory genes. Sbf1 may participate in bloodtestis barrier formation and recycling (Mruk \& Cheng 2011). The functional blood-testis barrier precludes the immune system attacking the shielded germ cells, but in the mutant, they can become more exposed. As IL6 and its receptor are involved in autoimmune orchitis (Rival et al. 2006), this mechanism could be plausible. However, as TLR8 and IL6R are abundant in leukocytes, it can also only be an increased relative blood amount in the mutant testis due to lower spermatid number or some other anomaly. Involvement of cellular junctions between Sertoli cells and between Sertoli and germ cells in the pathogenesis of male infertility in the rat Sbf1 mutants is also corroborated by the finding of $\beta 1$-integrin transcripts upregulation, Sertoli cell vacuolization and disorganization of the seminiferous epithelium with spermatocytes in adluminal compartment in mutants. Interestingly, $\beta 1$-integrin (Itgb1) and also Testin transcripts were upregulated as a response to testicular toxicity of 1,3-dinitrobenzene (Matsuyama et al. 2011), suggesting common mechanisms of Sertoli cell dysfunction.

In conclusion, we identified a truncating mutation of Sbf1 in the rat that recapitulates exactly spermatogenic arrest and azoospermia in the Sbf1 null mice, confirming thus the necessary role of Sbf1 in mammalian spermatogenesis.

\section{Supplementary data}

This is linked to the online version of the paper at http://dx.doi. org/10.1530/REP-16-0042.

\section{Declaration of interest}

The authors declare that there is no conflict of interest that could be perceived as prejudicing the impartiality of the research reported.

\section{Funding}

This work was supported by the Ministry of Health of the Czech Republic grant No. NT/12269 (F L) and the Czech Science Foundation grant No. 16-06548S (M P).

\section{References}

Agarwal A, Mulgund A, Hamada A \& Chyatte MR 2015 A unique view on male infertility around the globe. Reproductive Biology and Endocrinology 13 37. (doi:10.1186/s12958-015-0032-1)

Alazami AM, Alzahrani F, Bohlega S \& Alkuraya FS 2014 SET binding factor 1 (SBF1) mutation causes Charcot-Marie-tooth disease type 4B3. Neurology 82 1665-1666. (doi:10.1212/WNL.0000000000000331)
Beardsley A, Robertson DM \& O'Donnell L 2006 A complex containing alpha6beta1-integrin and phosphorylated focal adhesion kinase between Sertoli cells and elongated spermatids during spermatid release from the seminiferous epithelium. Journal of Endocrinology 190 759-770. (doi:10.1677/joe.1.06867)

Benson G 1999 Tandem repeats finder: a program to analyze DNA sequences. Nucleic Acids Research 27 573-580. (doi:10.1093/ nar/27.2.573)

Bohlega S, Alazami AM, Cupler E, Al-Hindi H, Ibrahim E \& Alkuraya FS 2011 A novel syndromic form of sensory-motor polyneuropathy is linked to chromosome 22q13.31-q13.33. Clinical Genetics 79 193-195. (doi:10.1111/cge.2011.79.issue-2)

Boivin J, Bunting L, Collins JA \& Nygren KG 2007 International estimates of infertility prevalence and treatment-seeking: potential need and demand for infertility medical care. Human Reproduction 22 1506-1512. (doi:10.1093/humrep/dem046)

Bolino A, Bolis A, Previtali SC, Dina G, Bussini S, Dati G, Amadio S, Del Carro U, Mruk DD, Feltri ML et al. 2004 Disruption of Mtmr2 produces CMT4B1-like neuropathy with myelin outfolding and impaired spermatogenesis. Journal of Cell Biology 167 711-721. (doi:10.1083/ jcb.200407010)

Brown PR, Miki K, Harper DB \& Eddy EM 2003 A-kinase anchoring protein 4 binding proteins in the fibrous sheath of the sperm flagellum. Biology of Reproduction 68 2241-2248. (doi:10.1095/ biolreprod.102.013466)

Cheng CY, Grima J, Stahler MS, Lockshin RA \& Bardin CW 1989 Testins are structurally related Sertoli cell proteins whose secretion is tightly coupled to the presence of germ cells. Journal of Biological Chemistry 264 21386-21393.

Dasari P, Nicholson IC \& Zola H 2008 Toll-like receptors. Journal of Biological Regulators and Homeostatic Agents 22 17-26.

Firestein R, Nagy PL, Daly M, Huie P, Conti M \& Cleary ML 2002 Male infertility, impaired spermatogenesis, and azoospermia in mice deficient for the pseudophosphatase Sbf1. Journal of Clinical Investigation 109 1165-1172. (doi:10.1172/jci0212589)

Foresta C, Moro E \& Ferlin A 2001 Y chromosome microdeletions and alterations of spermatogenesis. Endocrine Reviews 22 226-239. (doi:10.1210/er.22.2.226)

Golden WL, von Kap-Herr C, Kurth B, Wright RM, Flickinger CJ, Eddy R, Shows T \& Herr JC 1993 Refinement of the localization of the gene for human intraacrosomal protein SP-10 (ACRV1) to the junction of bands q23-->q24 of chromosome 11 by nonisotopic in situ hybridization. Genomics 18 446-449. (doi:10.1006/geno.1993.1496)

Hotaling J \& Carrell DT 2014 Clinical genetic testing for male factor infertility: current applications and future directions. Andrology 2 339-350. (doi:10.1111/andr.2014.2.issue-3)

Krausz C \& Chianese C 2014 Genetic testing and counselling for male infertility. Current Opinion in Endocrinology Diabetes and Obesity 21 244-250. (doi:10.1097/MED.0000000000000058)

Laporte J, Bedez F, Bolino A \& Mandel JL 2003 Myotubularins, a large disease-associated family of cooperating catalytically active and inactive phosphoinositides phosphatases. Human Molecular Genetics 12 R285-R292. (doi:10.1093/hmg/ddg273)

Liška F 2003 Selected genetic aspects of male infertility - what animal models tell us. Folia Biologica 49 129-141.

Lysiak JJ 2004 The role of tumor necrosis factor-alpha and interleukin-1 in the mammalian testis and their involvement in testicular torsion and autoimmune orchitis. Reproductive Biology and Endocrinology 29. (doi:10.1186/1477-7827-2-9)

Marchler-Bauer A, Derbyshire MK, Gonzales NR, Lu S, Chitsaz F, Geer LY, Geer RC, He J, Gwadz M, Hurwitz DI et al. 2015 CDD: NCBI's conserved domain database. Nucleic Acids Research 43 D222-D226. (doi:10.1093/nar/gku1221)

Matsuyama T, Niino N, Kiyosawa N, Kai K, Teranishi M \& Sanbuissho A 2011 Toxicogenomic investigation on rat testicular toxicity elicited by 1,3-dinitrobenzene. Toxicology 290 169-177. (doi:10.1016/j. tox.2011.09.001)

Mruk DD \& Cheng CY 2011 The myotubularin family of lipid phosphatases in disease and in spermatogenesis. Biochemical Journal 433 253-262. (doi:10.1042/BJ20101267)

Mulholland DJ, Dedhar S \& Vogl AW 2001 Rat seminiferous epithelium contains a unique junction (Ectoplasmic specialization) with signaling 
properties both of cell/cell and cell/matrix junctions. Biology of Reproduction 64 396-407. (doi:10.1095/biolreprod64.1.396)

Nakhro K, Park JM, Hong YB, Park JH, Nam SH, Yoon BR, Yoo JH, Koo H, Jung SC, Kim HL et al. 2013 SET binding factor 1 (SBF1) mutation causes Charcot-Marie-Tooth disease type 4B3. Neurology 81 165-173. (doi:10.1212/WNL.0b013e31829a3421)

Nam SY, Maeda S, Ogawa K, Kurohmaru M \& Hayashi Y 1997 Expression pattern of the mitochondrial capsule selenoprotein mRNA in the mouse testis after puberty; in situ hybridization study. Journal of Veterinary Medical Science 59 983-988. (doi:10.1292/jvms.59.983)

Nicot AS \& Laporte J 2008 Endosomal phosphoinositides and human diseases. Traffic 9 1240-1249. (doi:10.1111/tra.2008.9.issue-8)

Payne AH \& Youngblood GL 1995 Regulation of expression of steroidogenic enzymes in Leydig cells. Biology of Reproduction 52 217-225. (doi:10.1095/biolreprod52.2.217)

Reese MG, Eeckman FH, Kulp D \& Haussler D 1997 Improved splice site detection in Genie. Journal of Computational Biology 4 311-323. (doi:10.1089/cmb.1997.4.311)

Rival C, Theas MS, Guazzone VA \& Lustig L 2006 Interleukin-6 and IL-6 receptor cell expression in testis of rats with autoimmune orchitis. Journal of Reproductive Immunology 70 43-58. (doi:10.1016/j.jri.2005.10.006)

Untergasser A, Cutcutache I, Koressaar T, Ye J, Faircloth BC, Remm M \& Rozen SG 2012 Primer3 - new capabilities and interfaces. Nucleic Acids Research 40 e115. (doi:10.1093/nar/gks596)
Vincent MC, Daudin M, De MP, Massat G, Mieusset R, Pontonnier F, Calvas P, Bujan L \& Bourrouillout G 2002 Cytogenetic investigations of infertile men with low sperm counts: a 25-year experience. Journal of Andrology 23 18-22.

Welch JE, Brown PR, O'Brien DA \& Eddy EM 1995 Genomic organization of a mouse glyceraldehyde 3-phosphate dehydrogenase gene (Gapd-s) expressed in post-meiotic spermatogenic cells. Developmental Genetics 16 179-189. (doi:10.1002/(ISSN)1520-6408)

Yang K, Meinhardt A, Zhang B, Grzmil P, Adham IM \& Hoyer-Fender S 2012 The small heat shock protein ODF1/HSPB10 is essential for tight linkage of sperm head to tail and male fertility in mice. Molecular and Cellular Biology 32 216-225. (doi:10.1128/MCB.06158-11)

Ye J, Coulouris G, Zaretskaya I, Cutcutache I, Rozen S \& Madden TL 2012 Primer-BLAST: a tool to design target-specific primers for polymerase chain reaction. BMC Bioinformatics 13 134. (doi:10.1186/1471-210513-134)

Received 23 January 2016

First decision 23 February 2016

Revised manuscript received 25 May 2016

Accepted 17 June 2016 